\title{
Harnwegsinfekte: Antibiotikaresistenzen vermeiden, Senföle nutzen
}

- Bei den urologisch relevanten Bakterien wie den gramnegativen E. coli hat sich die Resistenzsituation in den letzten Jahren zunehmend verschlechtert. Neben einer Steigerung der Resistenzhäufigkeit gegen Cotrimoxazol und Ciprofloxacin - den bei akuten Harnwegsinfekten am häufigsten verordne-

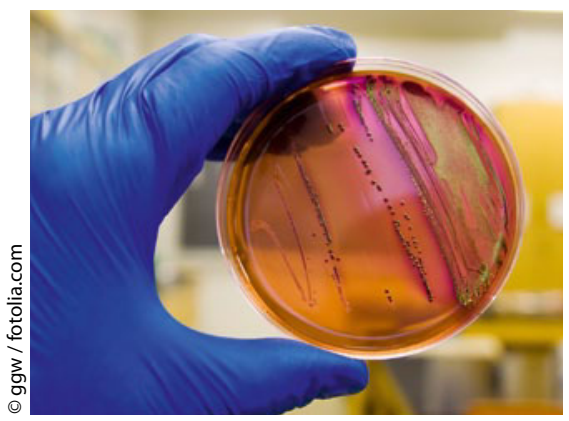

Immer mehr Bakterien entwickeln Resistenzen gegen Antibotika. ten Antibiotika - ist auch eine Zunahme von ESBL-bildenden („,extended spectrum betalactamases") Stämmen zu beobachten. Diese Bakterienstämme vermitteln ebenfalls eine Resistenz oder verminderte Empfindlichkeit gegenüber Cephalosporinen. Dementsprechend wird in den 2010 verabschiedeten S3Leitlinien der Deutschen Gesellschaft für Urologie empfohlen, Fluorchinolone und Cephalosporine bei der unkomplizierten Zystitis nicht als Antibiotika der ersten Wahl einzusetzen, solange es therapeutische Alternativen gibt. Wie Studien belegen, zeigen zum Beispiel die Senföle aus Kapuzinerkressenkraut und Meerrettichwurzel (ANGOCIN ${ }^{\circledR}$ Anti-Infekt N) bei akuten Harnwegsinfekten eine vergleichbare Wirksamkeit, bei einem erheblich besseren Verträglichkeits- und Sicherheitsprofil des pflanzlichen Präparates. Verschiedene In-vitro-Studien am Universitätsklinikum Freiburg zeigen ein breites anti- bakterielles Wirkspektrum der Senföle im grampositiven und gramnegativen Bereich, sogar gegen resistente Formen von E. coli und Problemkeime wie MRSA. Nach der neuesten Untersuchung gibt es keine wesentlichen Wirkungsunterschiede zwischen den multiresistenten und den korrespondierenden nicht resistenten Phänotypen. Dies bedeutet, dass die Senföle aus Kapuzinerkressenkraut und Meerrettichwurzel bei Nachweis von resistenten oder multiresistenten Erregern eine Behandlungsoption sein können. Auch der gramnegative Klebsiella pneumoniae reagiert auf das Pflanzengemisch. Berechnungen auf Basis des KrankenhausInfektions-Surveillance-Systems (KISS) zeigen, dass die Zahl der Intensivpatienten mit Klebsiella pneumoniae seit Jahren ansteigt.

Nach Informationen von

Repha, Langenhagen

\section{Nierenzellkarzinom: M-Tor-Inhibition überwindet Resistenzen}

\section{— Die Sequenztherapie mit zielgerichteten} Therapien ist beim fortgeschrittenen Nierenzellkarzinom (RCC) heute Standard. Aktuell wird dabei die optimale Reihenfolge an Substanzen diskutiert.

In der Erstlinientherapie werden in der Regel Tyrosinkinase-Inhibitoren (TKI) eingesetzt. Die Tumoren entwickeln jedoch nach einiger Zeit Resistenzmechanismen gegenüber gegen VEGF-gerichtete („,vascular endothelial growth factor") Behandlungen mit der Folge eines Progresses. Als Ursache für das Fortschreiten der Erkrankung gilt eine erworbene Resistenz des Tumors gegenüber dem angewandten Wirkmechanismus. In dieser Situation ist der Wechsel auf einen anderen Wirkmechanismus sinnvoll.

Mit Everolimus steht ein Präparat zur Verfügung, das die Serin/Threonin-Kinase mTOR inhibiert. Zudem greift der Wirkstoff komplex in die Signalwege der in Resistenzmechanismen involvierten Zellen ein: Tumor- und Endothelzellen, Perizyten und Fibroblasten.

Die Wirksamkeit von Everolimus nach Progress unter einer gegen VEGF-gerichteten Therapie wurde in der placebokontrollierten Phase-IIIStudie „RECORD-1" gezeigt (PFS: Everolimus vs. Placebo: 4,9 Monate vs. 1,9 Monate; $\mathrm{p}<$ $0,001)$. Aktuelle Ergebnisse einer Subgruppen- analyse dieser Zulassungsstudie weisen darauf hin, dass der oral verfügbare mTOR-Inhibitor nach der Therapie mit nur einem TKI ein längeres Ansprechen zeigt als nach zwei TKI. Auch wenn die Behandlung mit Everolimus beim fortgeschrittenen RCC wirksam und gut verträglich ist, entwickeln die Tumoren nach einiger Zeit ebenfalls Resistenzen gegenüber der mTOR-Inhibition. In experimentellen Tests in-vitro und in-vivo wurde nachgewiesen, dass die Resistenz gegenüber einer anti-angiogenen Behandlung mit der Anwendung des oralen mTOR-Inhibitors um- kehrbar ist. Resistenzmechanismen, die sich unter der TKI-Erstlinientherapie gebildet haben, können sich während der mTOR-Therapie zurückbilden. Wird in dieser Situation erneut ein TKI gegeben, zeigt sich häufig wieder eine anti-tumorale Aktivität. Der Wechsel auf Everolimus scheint somit eine Resensibilisierung auf anti-angiogene Substanzen zu ermöglichen. Mit der Sequenz TKI - Everolimus - TKI ist demnach ein therapeutischer Fortschritt in Sicht.

Nach Informationen von Novartis, Nürnberg

\section{Urologen helfen}

— Die Business Unit Urologie/Gynäkologie der Takeda Pharma, Tochter der japanischen Takeda Pharmaceutical Company Limited, hat in seiner groß angelegten Aktion „Urologen helfen" zu Spenden für die Betroffenen des Erdbebens in Japan Anfang März aufgerufen. Dank der langen, vertrauensvollen Zusammenarbeit mit den Medizinern konnte die Produktgruppe 50.000 EUR sammeln. Auch die Mitarbeiter von Takeda Pharma zeigten ihre Verbundenheit mit den Menschen in den Katastrophengebieten, indem sie zusammen 10.000 EUR spendeten. Takeda Pharma hat diese Summe von insgesamt 60.000 EUR verdoppelt, sodass von der deutschen Takeda-Niederlassung insgesamt 120.000 EUR an die Aktion Deutschland Hilft gehen konnten.

Nach Informationen von Takeda, Aachen 